\title{
Intonation and Compensation of Fretted String Instruments
}

\author{
Gabriele U. Varieschi and Christina M. Gower \\ Department of Physics, Loyola Marymount University - Los Angeles, CA 90045, USA*
}

\begin{abstract}
In this paper we present mathematical and physical models to be used in the analysis of the problem of intonation of musical instruments such as guitars, mandolins and the like, i.e., we study how to improve the tuning on these instruments.

This analysis begins by designing the placement of frets on the fingerboard according to mathematical rules and the assumption of an ideal string, but becomes more complicated when one includes the effects of deformation of the string and inharmonicity due to other string characteristics. As a consequence of these factors, perfect intonation of all the notes on the instrument can never be achieved, but complex compensation procedures are introduced and studied to minimize the problem.

To test the validity of these compensation procedures, we have performed extensive measurements using standard monochord sonometers and other basic acoustical devices, confirming the correctness of our theoretical models. In particular, these experimental activities can be easily integrated into standard acoustics courses and labs, and can become a more advanced version of basic experiments with monochords and sonometers.

PACS numbers: 01.55.+b; 01.50.Pa; 43.75.Bc; 43.75.Gh
\end{abstract}

Keywords: acoustics, musical acoustics, guitar, intonation, compensation

\footnotetext{
*Email: gvarieschi@lmu.edu; cgower@lion.lmu.edu
} 


\section{Contents}

I. Introduction

II. Geometrical model of a fretted string

A. Fret placement on the fingerboard

B. Deformation model of a fretted string

III. Compensation model

A. Vibrations of a stiff string

B. Compensation at nut and saddle

IV. Experimental measurements

A. Description of the apparatus

B. String properties and experimental results

V. Conclusions

\section{Acknowledgments}

\section{References}

\section{INTRODUCTION}

The physics of musical instruments is a very interesting sub-field of acoustics, which connects the mathematical models of vibrations and waves to the world of art and musical performance. This connection between science and music has always been present, since the origin of art and civilization. Classic books on the field are for example [1], [2], [3], 4]. In the 6th century B.C., the mathematician and philosopher Pythagoras was fascinated by music and by the intervals between musical tones. He was probably the first to perform experimental studies of the pitches of musical instruments and relate them to ratios of integer numbers.

This idea was the origin of the diatonic scale, which dominated much of western music, and also of the so-called just intonation system which was used for many centuries to tune musical instruments, based on perfect ratios of whole numbers. Eventually, this system was 
abandoned in favor of a more mathematically refined method for intonation and tuning, the well known equal temperament system, which was introduced by scholars such as Vincenzo Galilei (Galileo's father), Marin Mersenne and Simon Stevin, in the 16th and 17th centuries, and also strongly advocated by musicians such as the great J. S. Bach. In the equal-tempered scale, the interval of one octave is divided into 12 equal sub-intervals (semitones), achieving a more uniform intonation of musical instruments, especially when using all the 24 major and minor keys, as in Bach's masterpiece, the "Well Tempered Clavier." Historical discussion and complete reviews of the different intonation systems can be found in Refs. [5], [6], [7].

Mathematically, the twelve-tone equal temperament system requires the use of irrational numbers, since for example the ratio of the frequencies of two adjacent notes corresponds to $\sqrt[12]{2}$. On a fretted string instrument like a guitar, lute, mandolin, or similar, this intonation system is accomplished by placing the frets along the fingerboard according to these mathematical ratios. Unfortunately, even with the most accurate fret placement, perfect instrument tuning is never achieved. This is due mainly to the mechanical action of the player's fingers, which need to press the strings down on the fingerboard while playing, thus altering their length, tension and ultimately changing the frequency of the sound being produced. Other causes of imperfect intonation include inharmonicity of the strings, due to their intrinsic stiffness and other more subtle effects. An introduction to all these effects can be found in Refs. [8] and [9].

Experienced luthiers and guitar manufacturers usually correct for this effect by introducing the so-called compensation, i.e., they slightly increase the string length in order to compensate for the increased sound frequency, resulting from the effects described above (see instrument building techniques in [10], [11], [12], [13], [14]). Other solutions are reported in luthiers' websites ([15], [16], [17], [18], [19]) or in commercially patented devices ([20], [21], [22]). These empirical solutions can be improved by studying the problem in a more scientific fashion, through proper modeling of the string deformation and other effects, therefore leading to a new type of fret placement which is more effective for the proper intonation of the instrument.

Some mathematical studies of the problem appeared in specialized journals for luthiers and guitar builders ([23], [24]), but they were particularly targeted to luthiers and manufacturers of a specific instrument (typically classical guitar). We are not aware of similar scientific studies being reported in physics or acoustics publications. For example, in general 
physics journals we found only basic studies on guitar intonation and strings (see [25], [26], [27], [28], [29], [30], [31], [32], [33]), without any detailed analysis of the problem outlined above.

Therefore, our objective is to review and improve the existing mathematical models of compensation for fretted string instruments and to perform experimental measures to test these models. In particular, the experimental activities described in this paper were performed using standard lab equipment (sonometers and other basic acoustic devices) in view of the pedagogical goal of this project. In fact, all the experimental activities detailed in this work can be easily introduced in standard sound and waves lab courses, as an interesting variation of experiments usually performed with classic sonometers.

In the next section we will start by describing the geometry of the problem in terms of a simple string deformation model. In Sect. III we will examine the theoretical basis for the compensation model being used, and in Sect. IV we will describe the outcomes of our experimental activities.

\section{GEOMETRICAL MODEL OF A FRETTED STRING}

In this section we will introduce the geometrical model of a guitar fingerboard, review the practical laws for fret placement and study the deformations of a "fretted" string, i.e., when the string is pressed onto the fingerboard by the mechanical action of the fingers.

\section{A. Fret placement on the fingerboard}

We start our analysis by recalling Mersenne's law which describes the frequency $\nu$ of sound produced by a vibrating string [8], [9]:

$$
\nu_{n}=\frac{n}{2 L} \sqrt{\frac{T}{\mu}},
$$

where $n=1$ refers to the fundamental frequency, while $n=2,3 \ldots$ to the overtones. $L$ is the string length, $T$ is the tension, $\mu$ is the linear mass density of the string (mass per unit length), and $v=\sqrt{T / \mu}$ is the wave velocity.

In the equal-tempered musical scale an octave is divided into twelve semitones, mathematically: 


$$
\nu_{i}=\nu_{0} 2^{\frac{i}{12}} \simeq \nu_{0}(1.05946)^{i},
$$

where $\nu_{0}$ and $\nu_{i}$ are respectively the frequencies of the first note in the octave and of the $i$-th note $(i=1,2, \ldots, 12)$. For $i=12$, we obtain a frequency which is double that of the first note, as expected. Since Mersenne's law states that the fundamental frequency of the vibrating string is inversely proportional to the string length $L$, we simply combine Eqs. (1) and (2) to determine the correct string lengths for all the different notes $(i=1,2,3, \ldots)$ as a function of the original string length $L_{0}$ (open string length, producing the first note of the octave considered), assuming that the tension $T$ and the mass density $\mu$ are kept constant:

$$
L_{i}=L_{0} 2^{-\frac{i}{12}} \simeq L_{0}(0.943874)^{i} .
$$

This equation can be immediately used to determine the fret placement on a guitar or a similar instrument, since the frets essentially subdivide the string length into the required sub-lengths.

In Figure 1 we show a picture of a classical guitar as a reference. The string length is the distance between the saddle ${ }^{1}$ and the nut, while the frets are placed on the fingerboard at appropriate distances. We prefer to use the coordinate $X$, as illustrated in the same figure, to denote the position of the frets, measured from the saddle toward the nut position. $X_{0}$ will denote the position of the nut (the "zero" fret), while $X_{i}, i=1,2, \ldots$, are the positions of the frets of the instrument. On a classical guitar there are usually up to $19-20$ frets on the fingerboard and they are realized by inserting thin pieces of a special metal wire in the fingerboard, so that the frets will rise about $1.0-1.5 \mathrm{~mm}$ above the fingerboard level.

The positioning of the frets follows Eq. (3), which we rewrite in terms of our new variable $X$ :

$$
X_{i}=X_{0} 2^{-\frac{i}{12}} \simeq X_{0}(0.943874)^{i} \simeq X_{0}\left(\frac{17}{18}\right)^{i}
$$

\footnotetext{
${ }^{1}$ The saddle is the white piece of plastic or other material located near the bridge, on which the strings are resting. The strings are usually attached to the bridge, which is located on the left of the saddle. On other type of guitars, or other fretted instruments, the strings are attached directly to the bridge (without using any saddle). In this case the string length would be the distance between the bridge and the nut. Our analysis would not be different in this case: the bridge position would simply replace the saddle position.
} 


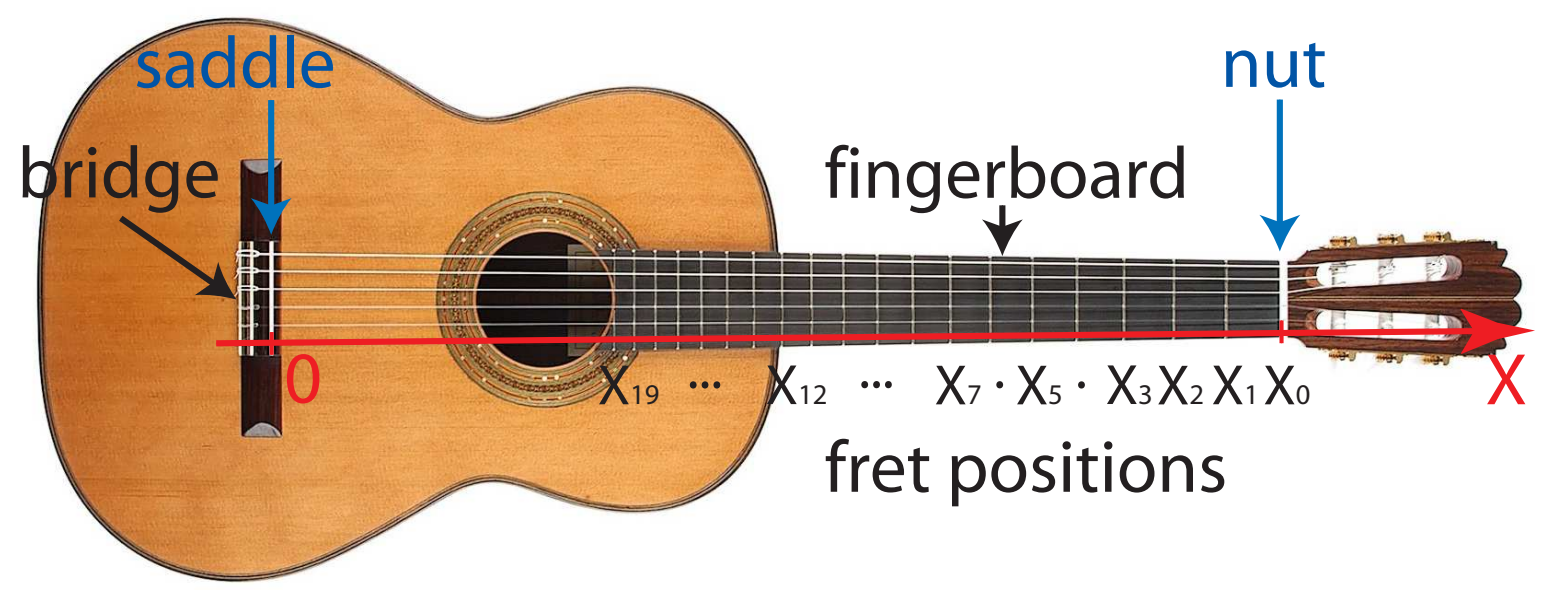

\section{Classical Guitar}

FIG. 1: Illustration of a classical guitar showing our coordinate system, from the saddle toward the nut, used to measure the fret positions on the fingerboard (guitar by Michael Peters - photo by Trilogy Guitars, reproduced with permission).

where the last approximation in the previous equation is the one historically employed by luthiers to practically locate the fret positions. This is usually called the "rule of 18," which requires placing the first fret at a distance from the nut corresponding to $\frac{1}{18}$ of the string length (or $\frac{17}{18}$ from the saddle); then place the second fret at a distance from the first fret corresponding to $\frac{1}{18}$ of the remaining length between the first fret and the saddle, and so on. Since $\frac{17}{18}=0.944444 \simeq 0.943874$, this empirical method is usually accurate enough for practical fret placement ${ }^{2}$, although modern luthiers use fret placement templates based on the decimal expression in Eq. (41).

\footnotetext{
${ }^{2}$ Following Eq. (4), frets number 5, 7, 12, and 19, are particularly important since they (approximately) correspond to vibrating string lengths which are respectively $3 / 4,2 / 3,1 / 2$, and $1 / 3$ of the full length, in line with the Pythagorean original theory of monochords.
} 


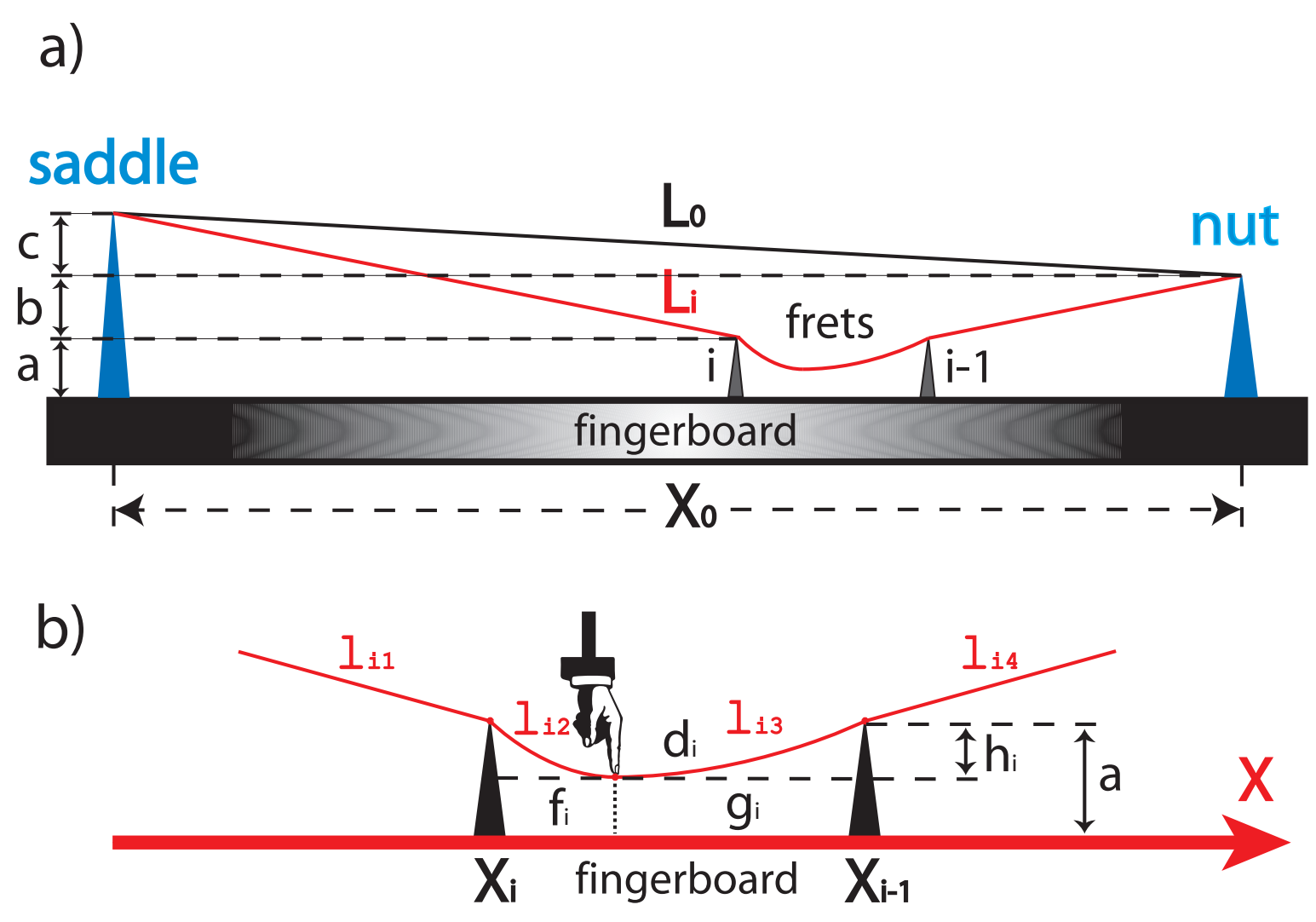

FIG. 2: Geometrical deformation model of a guitar string. In part a) we show the original string in black (of length $L_{0}$ ) and the deformed string in red (of length $L_{i}$ ) when it is pressed between frets $i$ and $i-1$. In part b) we show the details of our deformation model, in terms of the four different sub-lengths $l_{i 1}-l_{i 4}$ of the deformed string.

\section{B. Deformation model of a fretted string}

Figure 2 illustrates the geometrical model of a fretted string, i.e., when a player's finger or other device is pressing the string down to the fingerboard, until the string is resting on the desired $i-t h$ fret, thus producing the $i-t h$ note when the string is plucked. In this figure we use a notation similar to the one developed in Refs. [23], [24], but we will introduce a different deformation model.

Figure 2a shows the general geometrical variables for a guitar string. The distance $X_{0}$ 
between the saddle and the nut is also called the scale-length of the guitar (typically between $640-660 \mathrm{~mm}$ for a modern classical guitar) but this is not exactly the same as the real string length $L_{0}$, because saddle and nut usually have slightly different heights above the fingerboard surface. The connection between $L_{0}$ and $X_{0}$ is simply:

$$
L_{0}=\sqrt{X_{0}^{2}+c^{2}}
$$

The metal frets rise above the fingerboard by a distance $a$ as shown in Figure 2. The heights of the nut and saddle above the top of the frets are labeled in Figure 2 as $b$ and $c$, respectively. All these heights are greatly exaggerated; they are usually small compared to the string length. The standard fret positions are again denoted by $X_{i}$ and, in particular, we show the situation where the string is pressed between frets $i$ and $i-1$, thus reducing the vibrating portion of the string to the part between the saddle and the $i-t h$ fret.

Figure $2 \mathrm{~b}$ shows the details of the deformation caused by the action of a finger between the two frets. Previous works ([23], [24]) modeled this shape simply as a sort of "knife-edge" deformation which is not quite comparable to the action of a fingertip. We improved on this point by assuming a more "rounded" deformation, considering a curved shape as in Figure 2b. The action of the finger depresses the string behind the $i-t h$ fret by an amount $h_{i}$ below the fret level (not necessarily corresponding to the full height $a$ ) and at a distance $f_{i}$, compared to the distance $d_{i}$ between consecutive frets.

In Sect. IV we will describe how to set all these parameters to the desired values with our experimental device and simulate all possible deformations of the string. It is necessary for our compensation model, described in the next section, to compute exactly the length of the deformed string for any fret value $i$. As shown in Fig. 2, the deformed length $L_{i}$ of the entire string is the sum of the lengths of the four different parts:

$$
L_{i}=l_{i 1}+l_{i 2}+l_{i 3}+l_{i 4}
$$

where these four sub-lengths can be evaluated from the geometrical parameters as follows: 


$$
\begin{aligned}
& l_{i 1}=\sqrt{\left(X_{0} 2^{-\frac{i}{12}}\right)^{2}+(b+c)^{2}} \\
& l_{i 2}=h_{i} \sqrt{1+\frac{f_{i}^{2}}{4 h_{i}^{2}}+\frac{f_{i}^{2}}{4 h_{i}} \ln \left[\frac{2 h_{i}}{f_{i}}\left(1+\sqrt{1+f_{i}^{2} / 4 h_{i}^{2}}\right)\right]} \\
& l_{i 3}=h_{i} \sqrt{1+\frac{g_{i}^{2}}{4 h_{i}^{2}}+\frac{g_{i}^{2}}{4 h_{i}} \ln \left[\frac{2 h_{i}}{g_{i}}\left(1+\sqrt{1+g_{i}^{2} / 4 h_{i}^{2}}\right)\right]} \\
& l_{i 4}=\sqrt{X_{0}^{2}\left(1-2^{-\frac{i-1}{12}}\right)^{2}+b^{2}} .
\end{aligned}
$$

In Eq. (77) the sub-lengths $l_{i 2}$ and $l_{i 3}$ were obtained by using a simple parabolic shape for the "rounded" deformation shown in Fig. 2b, due to the action of the player's fingertip. They were computed by integrating the length of the two parabolic arcs shown in Fig. 2b, in terms of the distances $f_{i}, g_{i}$ and $h_{i}$.

The distances between consecutive frets are calculated as:

$$
d_{i}=f_{i}+g_{i}=X_{i-1}-X_{i}=X_{0} 2^{-\frac{i}{12}}\left(2^{\frac{1}{12}}-1\right)
$$

so that, given the values of $X_{0}, a, b, c, h_{i}$ and $f_{i}$, we can compute for any fret number $i$ the values of all the other quantities and the deformed length $L_{i}$. We will see in the next section that the fundamental geometrical quantities of the compensation model are defined as:

$$
Q_{i}=\frac{L_{i}-L_{0}}{L_{0}}
$$

and they can also be computed for any fret $i$ using Eqs. (50) - (8) .

\section{COMPENSATION MODEL}

In this section we will describe the model used to compensate for the string deformation and for the inharmonicity of a vibrating string, basing our analysis on the work done by G. Byers $([16],[24])$.

\section{A. Vibrations of a stiff string}

Strings used in musical instruments are not perfectly elastic, but possess a certain amount

of "stiffness" or inharmonicity which affects the frequency of the sound produced. Mersenne's 
law in Eq. (1) needs to be modified to include this property of real strings, yielding the following result (see Ref. [34], chapter 4, section 16):

$$
\nu_{n} \simeq \frac{n}{2 L} \sqrt{\frac{T}{\rho S}}\left[1+\frac{2}{L} \sqrt{\frac{E S k^{2}}{T}}+\left(4+\frac{n^{2} \pi^{2}}{2}\right) \frac{E S k^{2}}{T L^{2}}\right],
$$

where we have rewritten the linear mass density of the string as $\mu=\rho S$ ( $\rho$ is the string density and $S$ the cross section area). The correction terms inside the square brackets are due to the string stiffness and related to the modulus of elasticity (or Young's modulus) $E$ and to the radius of gyration $k$ (equal to the string radius divided by two, for a simple unwound steel or nylon string). Following Ref. [34], we will use c.g.s. units in the rest of the paper and in all computations, except when quoting some geometrical parameters for which it will be more convenient to use millimeters.

The previous equation is an approximation valid for $\frac{E S k^{2}}{T L^{2}}<\frac{1}{n^{2} \pi^{2}}$, a condition which is usually met in practical situations ${ }^{3}$. When the stiffness factor $\frac{E S k^{2}}{T L^{2}}$ is negligible, Eq. (10) reduces to the original Eq. (11). On the contrary, when this factor increases and becomes important, the allowed frequencies also increase, following the last equation, and the overtones $(n=2,3, \ldots)$ increase in frequency more rapidly than the fundamental tone $(n=1)$. The sound produced is no longer "harmonic" since the overtone frequencies are no longer simple multiples of the fundamental one, as seen from Eq. (10). In addition, the deformation of the fretted string, described in the previous section, will alter the string length $L$ and, as a consequence of this effect, will also change the tension $T$ and the cross section $S$ in the last equation. These are the main causes of the intonation problem being studied. Additional causes that we cannot address in this work are the imperfections of the strings (non uniform cross section or density), the motion of the end supports (especially the saddle and the bridge, transmitting the vibrations to the rest of the instrument) which also changes the string length, the effects of friction, and others.

Following Byers [24] we define $\alpha_{n}=\left(4+\frac{n^{2} \pi^{2}}{2}\right)$ and $\beta=\sqrt{\frac{E S k^{2}}{T}}$, so that we can simplify Eq. (10):

\footnotetext{
3 The condition is equivalent to $n^{2}<\frac{1}{\pi^{2}} \frac{T L^{2}}{E S k^{2}} \approx 369 ; 803 ; 5052$, where the numerical values are related to the three steel strings we will use in Sect. IV (see string properties in Table I) and for the shortest possible vibrating length $L \simeq \frac{1}{3} L_{0} \simeq 21.5 \mathrm{~cm}$. The approximation in Eq. (10) is certainly valid for our strings, for at least $n \lesssim 19$.
} 


$$
\nu_{n} \simeq \frac{n}{2 L} \sqrt{\frac{T}{\rho S}}\left[1+2 \frac{\beta}{L}+\alpha_{n} \frac{\beta^{2}}{L^{2}}\right] .
$$

We then consider just the fundamental tone $(n=1)$ as being the frequency of the sound perceived by the human ear ${ }^{4}$ :

$$
\nu_{1} \simeq \frac{1}{2 L} \sqrt{\frac{T}{\rho S}}\left[1+2 \frac{\beta}{L}+\alpha \frac{\beta^{2}}{L^{2}}\right]
$$

where $\alpha=\alpha_{1}=\left(4+\frac{\pi^{2}}{2}\right)$ and $\beta$ is defined as above. In Eq. (12) $L$ represents the vibrating length of the string, which in our case is the length $l_{i 1}$ when the string is pressed down onto the $i-t h$ fret. To further complicate the problem, the quantities $T, S$ and $\beta$ in Eq. (12) depend on the actual total length of the string $L_{i}$, as computed in Eq. (66). In other words, we tune the open string, of original length $L_{0}$, at the appropriate tension $T$, but when the string is "fretted" its length is changed from $L_{0}$ to $L_{i}$, thus slightly altering the tension, the cross section, and also $\beta$ which is a function of the previous two quantities. This is the origin of the lack of intonation, common to all fretted instruments, which calls for an appropriate compensation mechanism, which will be analyzed in the next section.

\section{B. Compensation at nut and saddle}

The proposed solution [24] to the intonation problem is to adjust the fret positions to accommodate for the frequency changes described in the previous equation. The vibrating lengths $l_{i 1}$ are recomputed as $l_{i 1}^{\prime}=l_{i 1}+\Delta l_{i 1}$, where $\Delta l_{i 1}$ represents a small adjustment in the placement of the frets, so that the fundamental frequency from Eq. (12) will match the ideal frequency of Eq. (2) and the fretted note will be in tune.

The ideal frequency $\nu_{i}$ of the $i-t h$ note can be expressed by combining together Eqs. (2) and (12):

$$
\nu_{i}=\nu_{0} 2^{\frac{i}{12}} \simeq \frac{1}{2 L_{0}} \sqrt{\frac{T\left(L_{0}\right)}{\rho S\left(L_{0}\right)}}\left[1+2 \frac{\beta\left(L_{0}\right)}{L_{0}}+\alpha \frac{\left[\beta\left(L_{0}\right)\right]^{2}}{L_{0}^{2}}\right] 2^{\frac{i}{12}}
$$

\footnotetext{
4 This statement is also an approximation since the pitch (or perceived frequency) is affected by the presence of the overtones. See for example the discussion of the psychological characteristics of music in Olson [4].
} 
where all the quantities on the right-hand side of the previous equation are related to the open string length $L_{0}$, since $\nu_{0}$ is the frequency of the open string note. On the other hand, we can write the same frequency $\nu_{i}$ using Eq. (12) directly for the fretted note:

$$
\nu_{i} \simeq \frac{1}{2 l_{i 1}^{\prime}} \sqrt{\frac{T\left(L_{i}\right)}{\rho S\left(L_{i}\right)}}\left[1+2 \frac{\beta\left(L_{i}\right)}{l_{i 1}^{\prime}}+\alpha \frac{\left[\beta\left(L_{i}\right)\right]^{2}}{l_{i 1}^{\prime 2}}\right],
$$

where now we use the "adjusted" vibrating length $l_{i 1}^{\prime}$ for the fretted note and all the other quantities on the right-hand side of Eq. (14) depend on the fretted string length $L_{i}$. By comparing Eqs. (13) and (14) we obtain the master equation for our compensation model:

$$
\frac{1}{2 L_{0}} \sqrt{\frac{T\left(L_{0}\right)}{\rho S\left(L_{0}\right)}}\left[1+2 \frac{\beta\left(L_{0}\right)}{L_{0}}+\alpha \frac{\left[\beta\left(L_{0}\right)\right]^{2}}{L_{0}^{2}}\right] 2^{\frac{i}{12}}=\frac{1}{2 l_{i 1}^{\prime}} \sqrt{\frac{T\left(L_{i}\right)}{\rho S\left(L_{i}\right)}}\left[1+2 \frac{\beta\left(L_{i}\right)}{l_{i 1}^{\prime}}+\alpha \frac{\left[\beta\left(L_{i}\right)\right]^{2}}{l_{i 1}^{\prime 2}}\right] .
$$

We obtained an approximate solution ${ }^{5}$ of the previous equation by Taylor expanding the right-hand side in terms of $\Delta l_{i 1}$ and by solving the resulting expression for the new vibrating lengths $l_{i 1}^{\prime}$ :

$$
l_{i 1}^{\prime} \simeq l_{i 1}\left\{1+\frac{\left[1+\frac{2 \beta\left(L_{0}\right)}{l_{i 1}}+\frac{\alpha\left[\beta\left(L_{0}\right)\right]^{2}}{l_{i 1}^{2}}\right]-\frac{1}{\left[1+Q_{i}(1+R)\right]}\left[1+\frac{2 \beta\left(L_{0}\right)}{L_{0}}+\frac{\alpha\left[\beta\left(L_{0}\right)\right]^{2}}{L_{0}^{2}}\right]}{\left[1+\frac{4 \beta\left(L_{0}\right)}{l_{i 1}}+\frac{3 \alpha\left[\beta\left(L_{0}\right)\right]^{2}}{l_{i 1}^{2}}\right]}\right\} .
$$

In this equation the quantities $Q_{i}$ are derived from Eq. (9) and from our new deformation model described in Sect. IIB, while an additional experimental quantity $R$ is introduced in the previous equation and defined as (see Ref. [24] for details):

$$
R=\left[\frac{d \nu}{d L}\right]_{L_{0}} \frac{L_{0}}{\nu_{0}}
$$

i.e., the frequency change $d \nu$ relative to the original frequency $\nu_{0}$, induced by an infinitesimal string length change $d L$, relative to the original string length $L_{0}$. This quantity will be measured in Sect. IV for the strings we used in this project.

The new vibrating lengths $l_{i 1}^{\prime}$ from Eq. (16) correspond to new fret positions $X_{i}^{\prime}$, since $X_{i}^{\prime}=\sqrt{l_{i 1}^{\prime 2}-(b+c)^{2}} \simeq l_{i 1}^{\prime}$ for $(b+c) \ll l_{i 1}^{\prime}$. A similar relation also holds between $X_{i}$ and

\footnotetext{
${ }^{5}$ Our solution in Eq. (16) differs from the similar solution obtained by Byers et al. (Eq. 17 in Ref. [24]). We believe that this is due to a minor error in their computation, which yields only minimal changes in the numerical results. Therefore, the compensation procedure used by G. Byers in his guitars is essentially correct and practically very effective in improving the intonation of his instruments.
} 
$l_{i 1}$ (see Fig. 2) so that the same Eq. (16) can be used to determine the new fret positions from the old ones:

$$
X_{i}^{\prime} \simeq X_{i}\left\{1+\frac{\left[1+\frac{2 \beta\left(L_{0}\right)}{l_{i 1}}+\frac{\alpha\left[\beta\left(L_{0}\right)\right]^{2}}{l_{i 1}^{2}}\right]-\frac{1}{\left[1+Q_{i}(1+R)\right]}\left[1+\frac{2 \beta\left(L_{0}\right)}{L_{0}}+\frac{\alpha\left[\beta\left(L_{0}\right)\right]^{2}}{L_{0}^{2}}\right]}{\left[1+\frac{4 \beta\left(L_{0}\right)}{l_{i 1}}+\frac{3 \alpha\left[\beta\left(L_{0}\right)\right]^{2}}{l_{i 1}^{2}}\right]}\right\}
$$

At this point a luthier should position the frets on the fingerboard according to Eq. (18) which is not anymore in the canonical form of the original Eq. (4). Moreover, each string would get slightly different fret positions, since the physical properties such as tension, cross section, etc., are different for the various strings of a musical instrument. Therefore, this compensation solution would be very difficult to be implemented practically and would also affect the playability of the instrument ${ }^{6}$.

An appropriate compromise, also introduced by Byers [24], is to fit the new fret positions $\left\{X_{i}^{\prime}\right\}_{i=1,2, \ldots}$ to a canonical fret position equation (similar to the original Eq. (4) ) of the form:

$$
X_{i}^{\prime}=X_{0}^{\prime} 2^{-\frac{i}{12}}+\Delta S
$$

where $X_{0}^{\prime}$ is a new scale length for the string and $\Delta S$ is the "saddle setback," i.e., the distance by which the saddle position should be shifted from its original position (usually $\Delta S>0$ and the saddle is moved away from the nut). The nut position is also shifted, but we require to keep the string scale at the original value $X_{0}$, therefore we need $X_{N U T}^{\prime}+\Delta S=X_{0}$, where $X_{N U T}^{\prime}$ is the new nut position in the primed coordinates. Introducing the shift in the nut position $\Delta N$ as $X_{N U T}^{\prime}=X_{0}^{\prime}+\Delta N$ and combining together the last two equations, we obtain the definition of the "nut adjustment" $\Delta N$ as:

$$
\Delta N=X_{0}-\left(X_{0}^{\prime}+\Delta S\right)
$$

This is typically a negative quantity, indicating that the nut has to be moved slightly forward toward the saddle.

Finally, instead of adopting a new scale length $X_{0}^{\prime}$, the luthier might want to keep the same original scale length $X_{0}$ and keep the fret positions according to the original Eq. (4)).

\footnotetext{
${ }^{6}$ Nevertheless some luthiers actually construct guitars where the individual frets under each string are adjustable in position by moving them slightly along the fingerboard. Each note of the guitar is then individually fine-tuned to achieve the desired intonation, requiring a very time consuming tuning procedure.
} 
Since the corrections and the effects we described above are essentially all linear with respect to the scale length adopted, it will be sufficient to rescale the nut and saddle adjustment as follows:

$$
\begin{aligned}
\Delta S_{\text {resc }} & =\frac{X_{0}}{X_{0}^{\prime}} \Delta S \\
\Delta N_{\text {resc }} & =\frac{X_{0}}{X_{0}^{\prime}} \Delta N .
\end{aligned}
$$

This final rescaling is also practically needed on a guitar or other fretted instrument, because the compensation procedure described above has to be carried out independently on each string of the instrument, i.e., all the quantities in the equations of this sections should be rewritten adding a string index $j=1,2, \ldots, 6$, for the six guitar strings. Each string would get a particular saddle and nut correction, but once these corrections are all rescaled according to Eq. (21) the luthier can still set the frets according to the original Eq. (4). The saddle and nut will be shaped in a way to incorporate all the saddle-nut compensation adjustments for each string of the instrument (see [24], 16] for practical illustrations of these techniques).

In practice, this compensation procedure does not change the original fret placement and the scale length of the guitar, but requires very precise nut and saddle adjustments for each of the strings of the instrument, using Eq. (21). Again, this is just a convenient approximation of the full compensation procedure, which would require repositioning all frets according to Eq. (18), but this would not be a very practical solution.

In the next section we will detail the experimental measures we performed following the deformation and compensation models outlined above. Since all our measurements will be carried out using a monochord apparatus, we will work essentially with a single string and not a whole set of six strings, as in a real guitar. Therefore, we will use all the equations outlined above without adding the additional string index $j$. However, it would be easy to modify our discussion in order to extend the deformation-compensation model to the case of a multi-string apparatus. 


\section{EXPERIMENTAL MEASUREMENTS}

In Figure 3 we show the experimental setup we used for our measurements. Since our goal was simply to test the physics involved in the intonation problem and not to build musical instruments or improve their construction techniques, we used standard lab equipment, as illustrated in the figure.

\section{A. Description of the apparatus}

A standard PASCO sonometer WA-9613 [35] was used as the main device for the experiment. This apparatus includes a set of steel strings of known linear density and diameter, and two adjustable bridges which can be used to simulate nut and saddle of a guitar. The string tension can be measured by using the sonometer tensioning lever, or adjusted directly with the string tensioning screw (on the left of the sonometer, as seen in Fig. 3). In particular, this adjustment allowed the direct measurement for each string of the $R$ parameter in Eq. (17), by slightly stretching the string and measuring the corresponding frequency change.

On top of the sonometer we placed a piece of a classical guitar fingerboard with scale length $X_{0}=645 \mathrm{~mm}$ (visible in Fig. 3 as a thin black object with twenty metallic frets, glued to a wooden board to raise it almost to the level of the string). The geometrical parameters

in Fig. 2 were set as follows: $a=1.3 \mathrm{~mm}$ (fret thickness), $b=1.5 \mathrm{~mm}, c=0.0 \mathrm{~mm}$ (since we used two identical sonometer bridges as nut and saddle). This arrangement ensured that the metal strings produced a good quality sound, without "buzzing" or producing undesired noise, when the sonometer was "played" like a guitar by gently plucking the string. Also, since we set $c=0$, the open string length is equal to the scale length: $L_{0}=X_{0}=645 \mathrm{~mm}$.

The mechanical action of the player's finger pressing on the string was produced by using a spring loaded device (also shown in Fig. 3, pressing between the sixth and seventh fret) with a rounded end to obtain the deformation model illustrated in Fig 2b. Although we tried different possible ways of pressing on the strings, for the measurements described in this section we were always pressing halfway between the frets $\left(f_{i}=g_{i}=\frac{1}{2} d_{i}\right)$ and all the way down on the fingerboard $\left(h_{i}=a=1.3 \mathrm{~mm}\right)$. In this way, all the geometrical parameters of Fig. 2 were defined and the fundamental quantities $Q_{i}$ of Eq. (9) could be computed. 


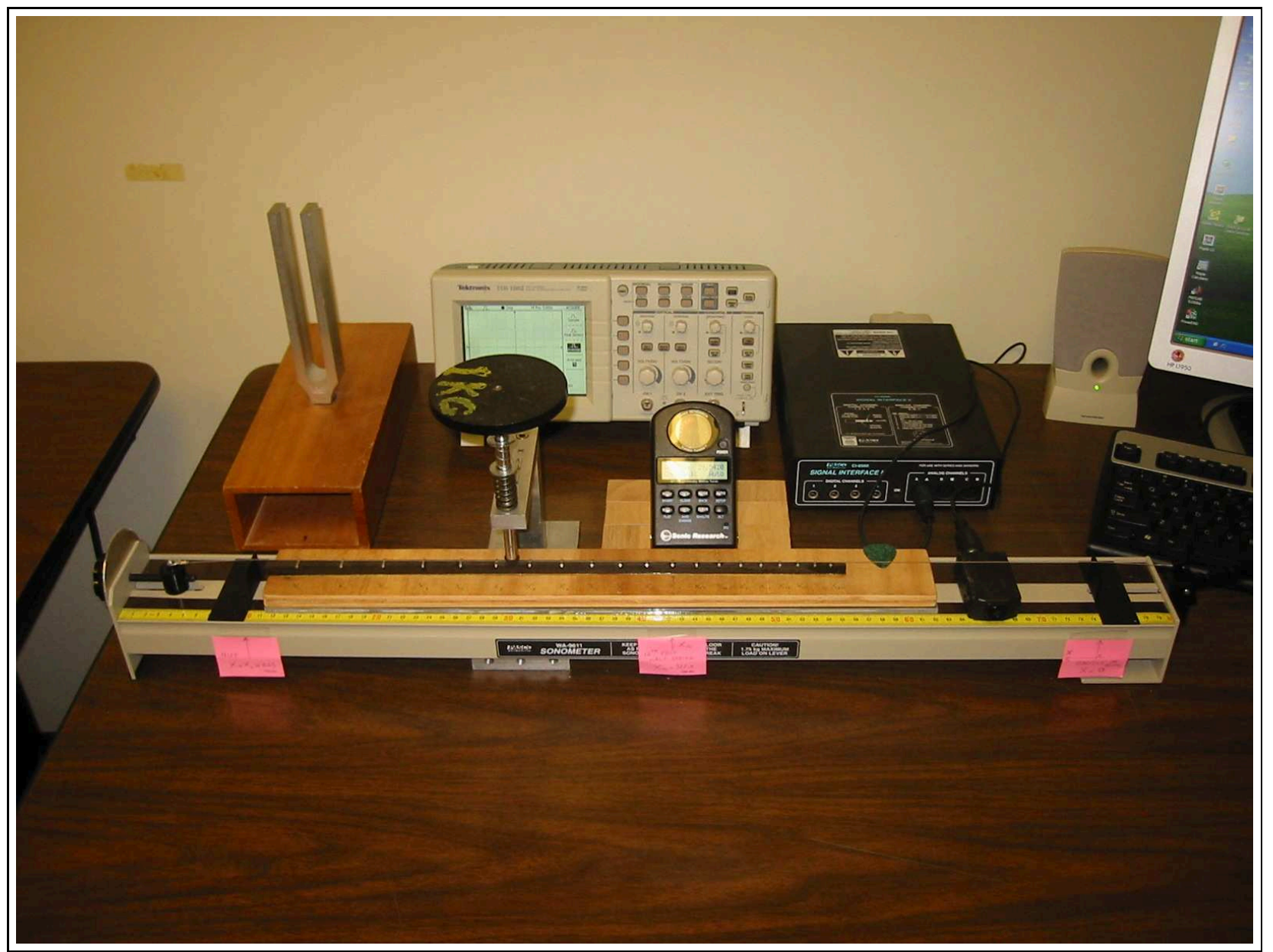

FIG. 3: Our experimental apparatus is composed of a standard sonometer to which we added a classical guitar fingerboard. Also shown is a mechanical device used to press the string on the fingerboard and several different instruments used to measure sound frequencies.

The sound produced by the plucked string (which was easily audible, due to the resonant body of the sonometer) was analyzed with different devices, in order to accurately measure its frequency. At first we used the sonometer detector coil or a microphone, connected to a digital oscilloscope, or alternatively to a computer through a digital signal interface, as shown also in Fig. 3. All these devices could measure frequencies in an accurate way, but we decided to use for most of our measurements a professional digital tuner [36], which could discriminate frequencies at the level of \pm 0.1 cents $^{7}$. This device is shown near the center

7 The cent is a logarithmic unit of measure used for musical intervals. The octave is divided into twelve semitones, each of which is subdivided in 100 cents, thus the octave is divided into 1200 cents. Since 


\begin{tabular}{||c|c|c|c||}
\hline \hline$\#$ & String 1 & String 2 & String 3 \\
\hline Open string note & $C_{3}$ & $F_{3}$ & $C_{4}$ \\
\hline Open string frequency $(H z)$ & 130.813 & 174.614 & 261.626 \\
\hline Radius $(\mathrm{cm})$ & 0.0254 & 0.0216 & 0.0127 \\
\hline Linear density $\mu(\mathrm{g} / \mathrm{cm})$ & 0.0150 & 0.0112 & 0.0039 \\
\hline Tension $($ dyne $)$ & $5.16 \times 10^{6}$ & $5.88 \times 10^{6}$ & $4.41 \times 10^{6}$ \\
\hline Young's modulus $E\left(d y n e / \mathrm{cm}^{2}\right)$ & $2.00 \times 10^{12}$ & $2.00 \times 10^{12}$ & $2.00 \times 10^{12}$ \\
\hline R parameter & 130 & 199 & 78.7 \\
\hline Rescaled saddle setback $\Delta S_{\text {resc }}(\mathrm{cm})$ & 0.733 & 0.998 & 0.518 \\
\hline Rescaled nut adjustment $\Delta N_{\text {resc }}(\mathrm{cm})$ & -2.31 & -2.41 & -1.35 \\
\hline \hline
\end{tabular}

TABLE I: The physical characteristics and the compensation parameters for the three steel strings used in our experimental tests are summarized here.

of Fig. [3, just behind the sonometer.

\section{B. String properties and experimental results}

For our experimental tests we chose three of the six steel guitar strings included with the PASCO sonometer. Their physical characteristics and the compensation parameters are described in Table 【.

The open string notes and related frequencies were chosen so that the sound produced using all the twenty frets of our fingerboard would span over 2-3 octaves, and the tensions were set accordingly. We used a value for Young's modulus which is typical of steel strings and we measured the $R$ parameter in Eq. (17) as explained in the previous section. The rescaled saddle setback $\Delta S_{\text {resc }}$ and the rescaled nut adjustment $\Delta N_{\text {resc }}$ from Eq. (21) were computed for each string, using the procedure outlined in Sect. III and the geometrical and physical parameters described above.

an octave corresponds to a frequency ratio of $2: 1$, one cent is precisely equal to an interval of $2 \frac{1}{1200}$. Given two frequencies $a$ and $b$ of two different notes, the number $n$ of cents between the notes is $n=$ $1200 \log _{2}(a / b) \simeq 3986 \log _{10}(a / b)$. Alternatively, given a note $b$ and the number $n$ of cents in the interval, the second note $a$ of the interval is $a=b \times 2^{\frac{n}{1200}}$. 
We then carefully measured the frequency of the sounds produced by pressing each string onto the twenty frets of the fingerboard in the two possible modes: without any compensation, i.e., setting the frets according to Eq. (4), and with compensation, i.e., after shifting the position of saddle and nut by the amounts specified in Table I and retuning the open string to the original note.

Table II illustrates the frequency values for String 1, obtained in the two different modes and compared to the theoretical values of the same notes for the case of a "perfect intonation" of the instrument. The measurements were repeated several times and the quantities in Table II] represent average values.

In this table, fret number zero represents the open string being plucked, so there is no difference in frequency for the three cases. On the contrary, for all the other frets, the frequencies without compensation are considerably higher than the theoretical values for a perfectly intonated instrument. This results in the pitch ${ }^{8}$ of these notes to be perceived being higher (or sharper) than the correct pitch. In fact, when we "played" our monochord sonometer in this first situation, it sounded definitely out of tune. The frequency values obtained instead by using our compensation correction appear to be much closer to the theoretical values, thus effectively improving the overall intonation of our monochord instrument.

In Table II we also show the frequency deviation of each note from the theoretical value of perfect intonation for both cases: with and without compensation. The frequency shifts are expressed in cents (see definition in note at the end of Sect. IVA) rather than in Hertz, since the former unit is more suitable to measure how the human ear perceives different sounds to be in tune or out of tune. The frequency deviation values illustrate more clearly the effectiveness of the compensation procedure: without compensation the deviation from perfect intonation ranges between 14.3 and 64.3 cents, with compensation this range is reduced to values between -7.9 and +7.3 cents.

In view of the previous discussion, we prefer to plot our results for String 1 in terms of the frequency deviation of each note from the theoretical value of perfect intonation.

\footnotetext{
${ }^{8}$ We note that the frequency of the sound produced is the physical quantity we measured in our experiments. The pitch is defined as a sensory characteristic arising out of frequency, but also affected by other subjective factors which depend upon the individual. It is beyond the scope of this paper to consider these additional subjective factors.
} 


\begin{tabular}{|c|c|c|c|c|c|c|}
\hline $\begin{array}{c}\text { String } 1 \\
\text { Fret } \\
\text { number }\end{array}$ & Note & $\begin{array}{c}\text { Perfect } \\
\text { intonation } \\
\text { Frequency } \\
(H z)\end{array}$ & $\begin{array}{c}\text { Without } \\
\text { compensation } \\
\text { Frequency } \\
(H z)\end{array}$ & $\begin{array}{c}\text { Without } \\
\text { compensation } \\
\text { Freq. deviation } \\
\text { (cents) }\end{array}$ & $\begin{array}{c}\text { With } \\
\text { compensation } \\
\text { Frequency } \\
(H z)\end{array}$ & $\begin{array}{c}\text { With } \\
\text { compensation } \\
\text { Freq. deviation } \\
\text { (cents) }\end{array}$ \\
\hline 0 & $C_{3}$ & 130.813 & 130.813 & 0 & 130.813 & 0 \\
\hline 1 & $C_{3}^{\#}$ & 138.591 & 143.832 & 64.3 & 137.958 & -7.9 \\
\hline 2 & $D_{3}$ & 146.832 & 150.551 & 43.3 & 147.323 & 5.8 \\
\hline 3 & $D_{3}^{\#}$ & 155.563 & 159.126 & 39.2 & 155.363 & -2.2 \\
\hline 4 & $E_{3}$ & 164.814 & 168.407 & 37.3 & 164.070 & -7.8 \\
\hline 5 & $F_{3}$ & 174.614 & 178.348 & 36.6 & 173.933 & -6.8 \\
\hline 6 & $F_{3}^{\#}$ & 184.997 & 188.754 & 34.8 & 184.763 & -2.2 \\
\hline 7 & $G_{3}$ & 195.998 & 200.386 & 38.3 & 195.878 & -1.1 \\
\hline 8 & $G_{3}^{\#}$ & 207.652 & 212.105 & 36.7 & 207.632 & -0.2 \\
\hline 9 & $A_{3}$ & 220.000 & 224.644 & 36.2 & 220.081 & 0.6 \\
\hline 10 & $A_{3}^{\#}$ & 233.082 & 237.495 & 32.5 & 233.136 & 0.4 \\
\hline 11 & $B_{3}$ & 246.942 & 252.345 & 37.5 & 247.123 & 1.3 \\
\hline 12 & $C_{4}$ & 261.626 & 266.338 & 30.9 & 261.505 & -0.8 \\
\hline 13 & $C_{4}^{\#}$ & 277.183 & 281.958 & 29.6 & 277.076 & -0.7 \\
\hline 14 & $D_{4}$ & 293.665 & 298.545 & 28.5 & 293.688 & 0.1 \\
\hline 15 & $D_{4}^{\#}$ & 311.127 & 315.276 & 22.9 & 311.463 & 1.9 \\
\hline 16 & $E_{4}$ & 329.628 & 334.822 & 27.1 & 329.787 & 0.8 \\
\hline 17 & $F_{4}$ & 349.228 & 353.408 & 20.6 & 348.785 & -2.2 \\
\hline 18 & $F_{4}^{\#}$ & 369.994 & 373.545 & 16.5 & 370.330 & 1.6 \\
\hline 19 & $G_{4}$ & 391.996 & 396.597 & 20.2 & 393.335 & 5.9 \\
\hline 20 & $G_{4}^{\#}$ & 415.305 & 418.742 & 14.3 & 417.068 & 7.3 \\
\hline
\end{tabular}

TABLE II: Frequency values of the different notes obtained with String 1: theoretical perfect intonation values are compared to the experimental values with and without compensation. Also shown are the frequency deviations (in cents) from the theoretical values, for both cases. 
Fig. 4 shows these frequency deviations for each fret number (corresponding to the different musical notes of Table II) in the two cases: without compensation (red circles) and with compensation (blue triangles). Error bars are also included, coming from the computed standard deviations of the measured frequency values.

We also show in the same figure the so-called pitch discrimination range (region between green dashed lines), i.e., the difference in pitch which an individual can effectively detect when hearing two different notes in rapid succession. In other words, notes within this range will not be perceived as different in pitch by human ears. It can be easily seen in Fig. 团 that all the (red) values without compensation are well outside the pitch discrimination range, thus will be perceived as out of tune (in particular as sharper sounds). On the contrary, the (blue) values with compensation are in general within the green dashed discrimination range of about \pm 10 cents $^{9}$. The compensation procedure has virtually made them equivalent to the perfect intonation values (corresponding to the zero cent deviation - perfect intonation level, black dotted line in Figure 44). We note again that fret number zero simply corresponds to playing the open string note which is always perfectly tuned, therefore the experimental points for this fret do not show any frequency deviation.

We repeated the same type of measurements also for String 2 and 3, which were tuned at higher frequencies as open strings (respectively as $F_{3}$ and $C_{4}$, see Table I). In this way we obtained experimental sets of measured frequencies, with and without compensation, for these two other strings, similar to those presented in Table I. For brevity, we will omit to report all these numerical values, but we present in Figs. 5 and 6 the frequency deviation plots, as we have done for String 1 in Fig. 4.

The results in Figs. [5 and [6 are very similar to those in the previous figure: the frequencies without compensation are much higher than the perfect intonation level, while the compensation procedure is able to reduce almost all the frequency values to the region within the green dashed curves (the pitch discrimination range). Using again the procedure outlined in Ref. [4], the discrimination ranges in Figs. [5 and 6] were computed respectively as \pm 8.6 cents and \pm 5.2 cents, due to the different frequencies produced by these two other

\footnotetext{
9 This discrimination range was estimated, for the frequenqies of String 1, according to the discussion in Ref. 4], pages 248-252. In general, this range varies from about \pm 5 cents - \pm 10 cents for frequencies between $1000-2000 \mathrm{~Hz}$, to even larger values of \pm 40 cents - \pm 50 cents at lower frequencies, between $60-120 \mathrm{~Hz}$.
} 


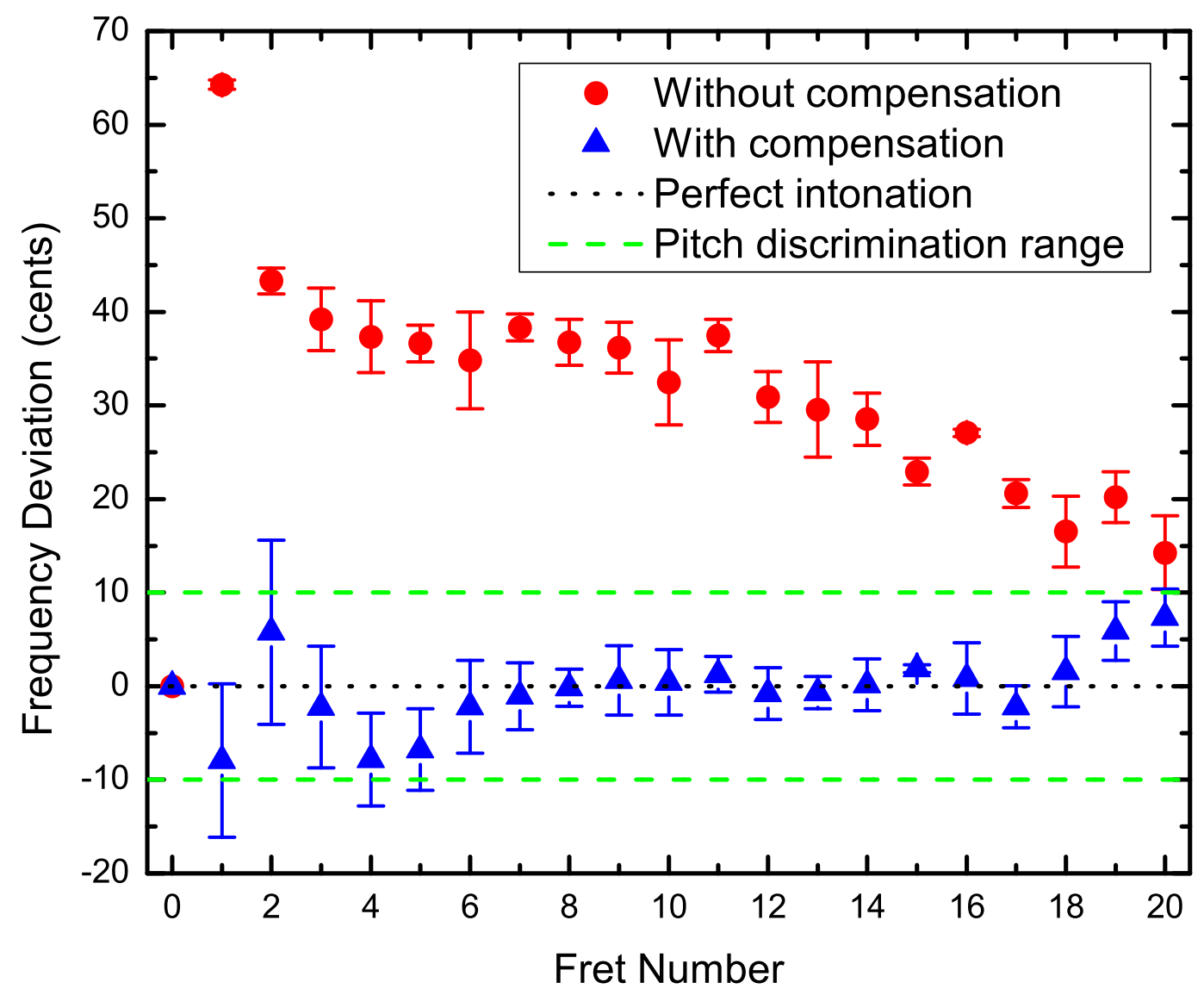

FIG. 4: Frequency deviation from perfect intonation level (black dotted line) for notes obtained with String 1. Red circles denote results without compensation, while blue triangles denote results with compensation. Also shown (region between green dashed lines) is the approximate pitch discrimination range for frequencies related to this string.

strings.

For the three cases we analyzed, we can conclude that the compensation procedure described in this paper is very effective in improving the intonation of each of the strings we used. Although more work on the subject is needed (in particular we need to test also nylon strings, which are more commonly used in classical guitars), we have proven that the intonation problem of fretted string instruments can be analyzed and solved using physical 


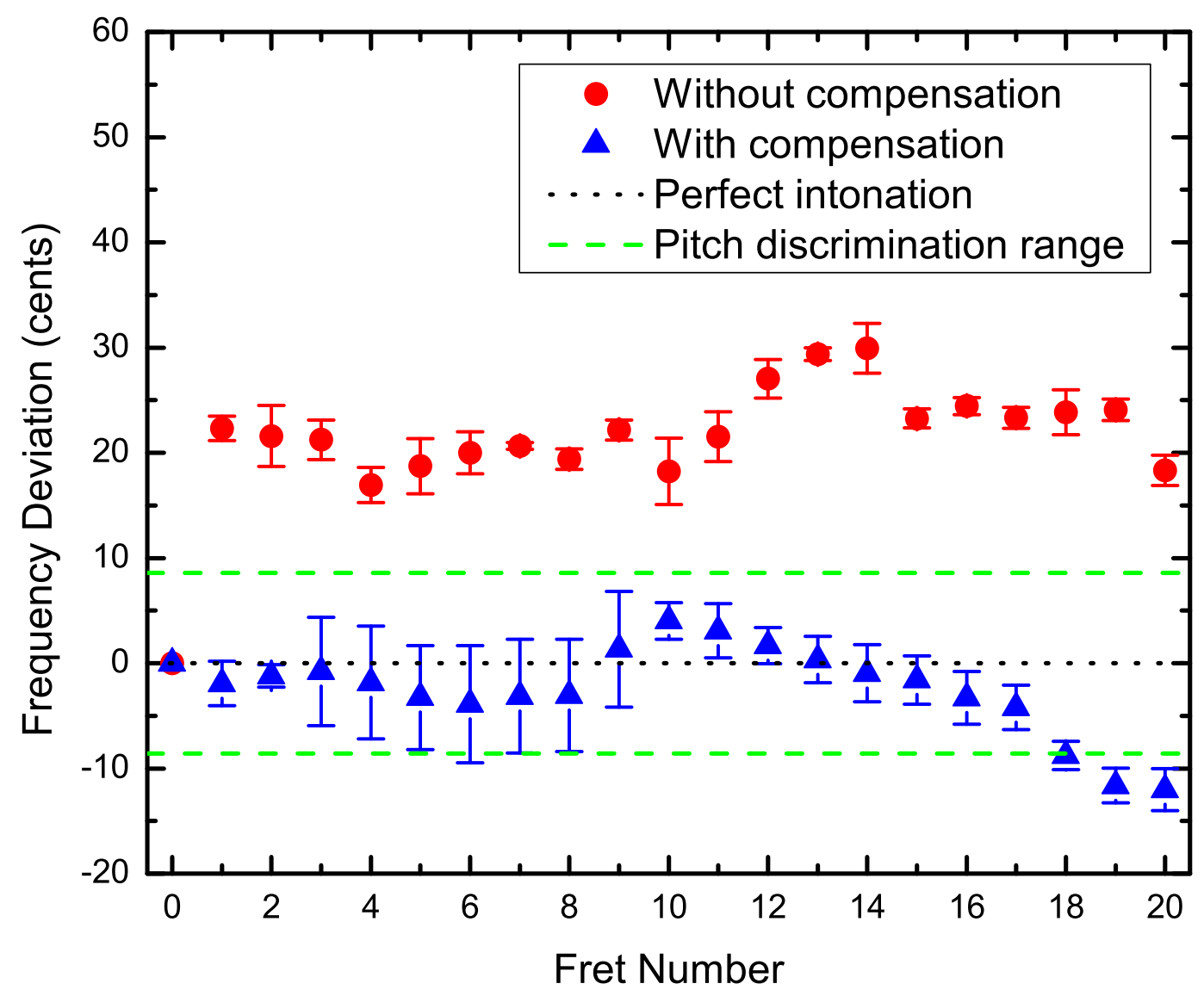

FIG. 5: Frequency deviation from perfect intonation level (black dotted line) for notes obtained with String 2. Red circles denote results without compensation, while blue triangles denote results with compensation. Also shown (region between green dashed lines) is the approximate pitch discrimination range for frequencies related to this string.

and mathematical models, which are more reliable than the empirical methods developed by luthiers during the historical development of these instruments. 


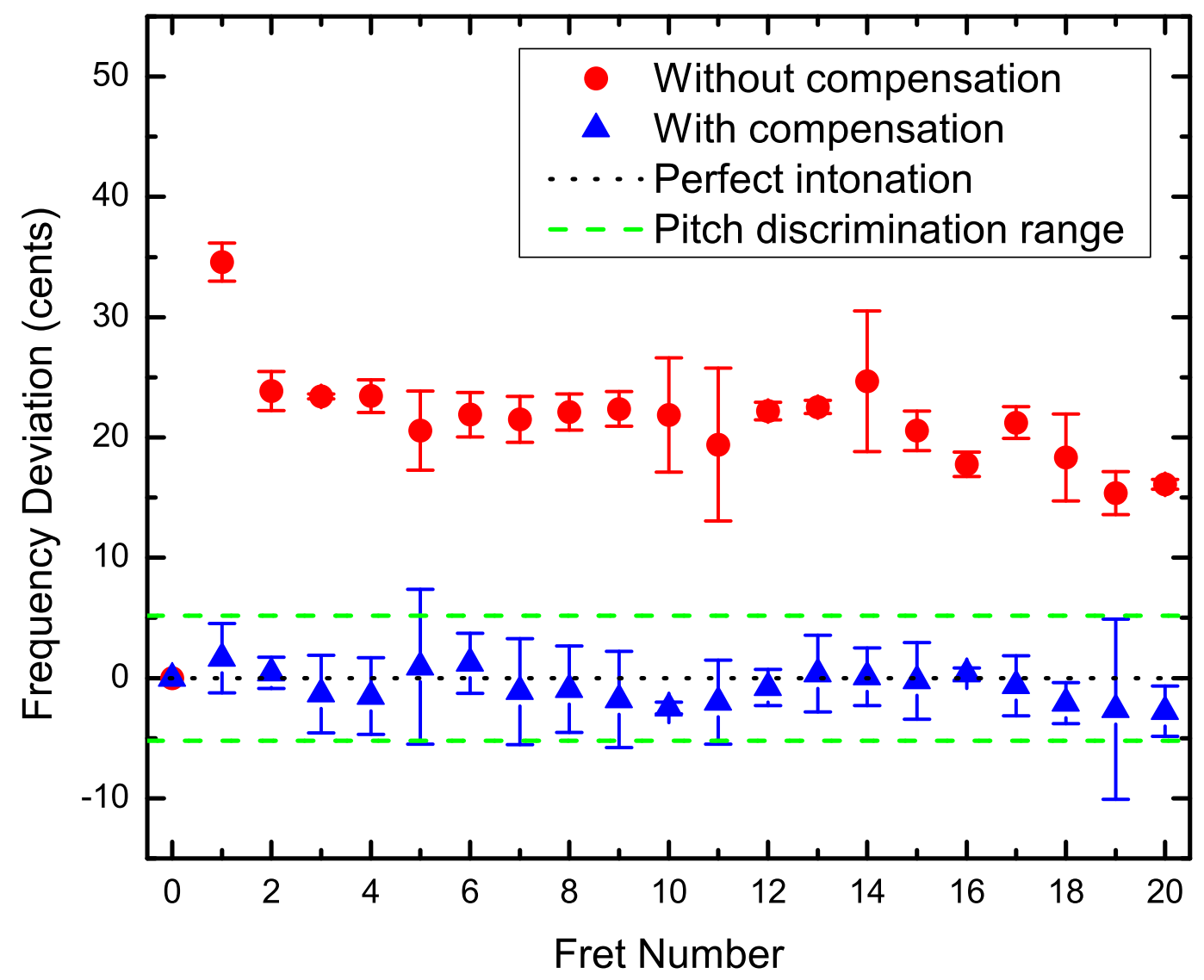

FIG. 6: Frequency deviation from perfect intonation level (black dotted line) for notes obtained with String 3. Red circles denote results without compensation, while blue triangles denote results with compensation. Also shown (region between green dashed lines) is the approximate pitch discrimination range for frequencies related to this string.

\section{CONCLUSIONS}

In this work we studied the mathematical models and the physics related to the problem of intonation and compensation of fretted string instruments. While this problem is usually solved in an empirical way by luthiers and instrument makers, we have shown that it is possible to find a mathematical solution, improving the original work by G. Byers and 
others, and that this procedure can be effectively implemented in practical situations.

We have demonstrated how to use simple lab equipment, such as standard sonometers and frequency measurement devices, to study the sounds produced by plucked strings, when they are pressed onto a guitar-like fingerboard, thus confirming the mathematical models for intonation and compensation. These activities can also be easily presented in standard musical acoustics courses, or used in sound and waves labs as an interesting variation of experiments usually performed with classic sonometers.

\section{Acknowledgments}

This work was supported by a grant from the Frank R. Seaver College of Science and Engineering, Loyola Marymount University. The authors would like to acknowledge useful discussions with John Silva of Trilogy Guitars and with luthier Michael Peters, who also helped us with the guitar fingerboard. We thank Jeff Cady for his technical support and help with our experimental apparatus. We are also very thankful to luthier Dr. Gregory Byers for sharing with us important details of his original study on the subject and other suggestions. Finally, the authors gratefully acknowledge the anonymous reviewers for their useful comments and suggestions.

[1] H. Helmoltz, On the Sensation of Tone (Dover Publications, New York, USA, 1954).

[2] J. Jeans, Science and Music (Dover Publications, New York, USA, 1968).

[3] A. H. Benade, Fundamentals of Musical Acoustics (Dover Publications, New York, USA, 1990).

[4] H. F. Olson, Music, Physics and Engineering (Dover Publications, New York, USA, 1967).

[5] S. Isacoff, Temperament (Vintage Books, New York, USA, 2001).

[6] J. M. Barbour, Tuning and Temperament - A Historical Survey (Dover Publications, Mineola, New York, USA, 2004).

[7] G. Loy, Musimatichs - The Mathematical Foundations of Music - Volume 1 (The MIT Press, Cambridge, Massachusetts, USA, 2006).

[8] F. R. M. Thomas D. Rossing and P. A. Wheeler, The Science of Sound (Addison Wesley, San 
Francisco, USA, 2002).

[9] N. H. Fletcher and T. D. Rossing, The Physics of Musical Instruments (Springer-Verlag, New York, USA, 1998).

[10] M. Rodriguez, The Art and Craft of Making Classical Guitars (Hal Leonard, Milwaukee, USA, 2003).

[11] B. Hopkin, Musical Instrument Design (See Sharp Press, Tucson, Arizona, USA, 1996).

[12] R. Middleton, The Guitar Maker's Workshop (The Crowood Press, Ramsbury, UK, 1997).

[13] W. R. Cumpiano and J. D. Natelson, Guitarmaking - Tradition and Technology (Chronicle Books, San Francisco, USA, 1993).

[14] R. Lundberg, Historical Lute Construction (Guild of American Luthiers, Tacoma, WA, USA, 2002).

[15] J. Gilbert and W. Gilbert, Intonation and Fret Placement (Webpage, http://www.schrammguitars.com/intonation.html).

[16] G. Byers, Intonation Research (Webpage, http://www.byersguitars.com/research/intonation.html).

[17] S. Stenzel, Classical Master Guitars (Webpage, http://www.stenzel-guitars.de).

[18] J. Locke, Intonation and tuning of the Classical Guitar (Webpage, http://www.classicalandflamencoguitars.com/Compensation.htm).

[19] M. Doolin, Intonation I-VIII (Webpage, http://www.doolinguitars.com/intonation/intonation1.html).

[20] R. S. Jones, US Patent N. 3,599,524 (1971).

[21] P. R. Smith, US Patent N. 4,295,404 (1981).

[22] H. B. Feiten, US Patent N. 5,814,745 (1998).

[23] W. Bartolini and P. Bartolini, Journal of Guitar Acoustics 6, 74 (1982).

[24] G. Byers, American Lutherie 47 (1996), 1995 GAL Convention.

[25] G. E. Jones and J. L. Ferguson, American Journal of Physics 48, 362 (1980).

[26] D. E. Hall, The Physics Teacher 27, 673 (1989).

[27] B. Saferstein, The Physics Teacher 29, 438 (1991).

[28] J. E. Smedley, American Journal of Physics 66, 144 (1998).

[29] M. C. LoPresto, Physics Education 38, 309 (2003).

[30] F. W. Inman, The Physics Teacher 44, 465 (2006).

[31] M. C. Lopresto, The Physics Teacher 44, 509 (2006).

[32] A. F. McKinley, The Physics Teacher 45, 4 (2007). 
[33] M. C. Lopresto, The Physics Teacher 46, 486 (2008).

[34] P. M. Morse, Vibration and Sound (American Institute of Physics, New York, USA, 1983).

[35] PASCO, WA-9613 Sonometer Instruction Manual (1988).

[36] TurboTuner, Model ST-122 True Strobe Tuner (Webpage, http://www.turbo-tuner.com). 